\title{
Discovery and Optimization of Two Eis Inhibitor Families as Kanamycin Adjuvants against Drug-Resistant M. tuberculosis
}

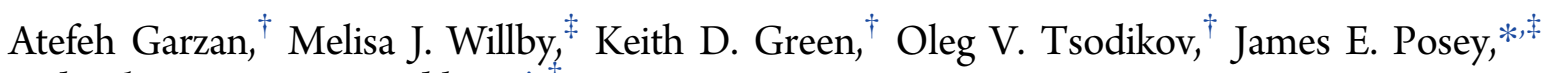 \\ and Sylvie Garneau-Tsodikova*,
}

${ }^{\dagger}$ Department of Pharmaceutical Sciences, College of Pharmacy, University of Kentucky, Lexington, Kentucky 40536, United States

${ }^{\ddagger}$ Mycobacteriology Laboratory Branch, Division of Tuberculosis Elimination, National Center for HIV/AIDS, Viral Hepatitis, STD, and TB Prevention, Centers for Disease Control and Prevention, Atlanta, Georgia 30333, United States

\section{Supporting Information}

ABSTRACT: Drug-resistant tuberculosis (TB) is a global threat and innovative approaches such as using adjuvants of anti-TB therapeutics are required to combat it. High-throughput screening yielded two lead scaffolds of inhibitors of Mycobacterium tuberculosis (Mtb) acetyltransferase Eis, whose upregulation causes resistance to the anti-TB drug kanamycin (KAN). Chemical optimization on these scaffolds resulted in potent Eis inhibitors. One compound restored the activity of KAN in a KAN-resistant $M t b$ strain. Model structures of Eisinhibitor complexes explain the structure-activity relationship.
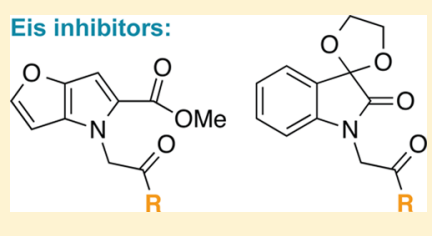

KEYWORDS: Aminoglycoside acetyltransferase, resistance, enzyme inactivation, drug combination, structure-activity relationship

$\mathrm{D}$ espite extensive efforts to discover new antitubercular agents in recent years, tuberculosis (TB) remains the top bacterial cause of mortality worldwide. The proportion of new multidrug-resistant (MDR)-TB cases has not changed in recent years. MDR strains of Mycobacterium tuberculosis $(M t b)$ are resistant to the first-line antituberculars isoniazid and rifampicin. Extensively drug-resistant (XDR) strains of $M t b$ are additionally resistant to fluoroquinolones and at least one of the second-line injectable anti-TB drugs, capreomycin, kanamycin (KAN), or amikacin. XDR-TB has very poor therapeutic outcomes. Thus, the discovery and development of new therapeutics is needed.

KAN is currently used to treat MDR- and XDR-Mtb infections. Resistance to KAN, observed in one-third of KAN-resistant infections, is due to the upregulation of the enhanced intracellular survival (Eis) protein in $M t b .{ }^{1}$ We previously established that Eis modifies aminoglycosides (AGs), ${ }^{2}$ capreomycin, ${ }^{3}$ and other lysine-containing biological molecules ${ }^{3}$ by a unique multiacetylating mechanism. ${ }^{4}$ We also reported crystal structures of Eis-CoA complexes and that of an Eis_Mtb-CoA-tobramycin complex. $^{5,6}$

New Eis inhibitors that can be used in combination with AGs such as KAN is a potential way to overcome the resistance due to Eis upregulation. We reported some structural scaffolds with inhibitory activity against the purified Eis enzyme in vitro. ${ }^{7}$ Here, we report two new scaffolds: the methyl $4 \mathrm{H}$-furo[3,2-b]pyrrole-5carboxylate (scaffold $\mathbf{1}$ ) and the 3-(1,3-dioxolano)-2-indolinone (scaffold 2) identified by high-throughput screening (HTS), the synthesis of 27 analogues of these scaffolds, and their biochemical and biological testing.

First, we screened $\sim 123,000$ small molecules for inhibition of KAN acetylation by Eis_Mtb. The HTS yielded two promising scaffolds 1 and 2 (Figure S1A). Sixty compounds containing scaffold 1 (Figure S2) were present in the HTS library. In these 60 compounds, the pyrrole ring was decorated with different groups including alkyl chains, aryl and oxazole rings, and amides. Fiftynine of these scaffold 1 molecules did not inhibit Eis $M t b$; however, compound 1a (Figure S1B and Scheme 1, also labeled as

Scheme 1. Preparation of Potential Eis Inhibitors: Scaffold 1 and Scaffold 2 Core Structures Generated in This Study

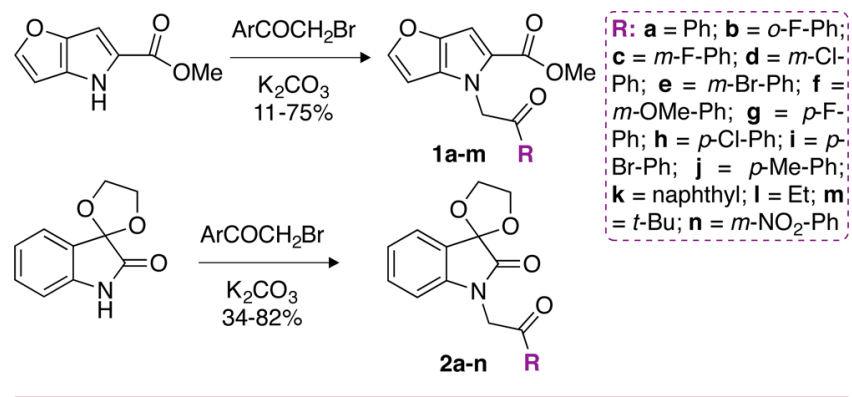

4kk in Figure S2) displayed some inhibition in the HTS. Because 1a contained an aryl ketone group, we opted to synthesize 1 a along with 12 additional analogues $(\mathbf{1} \mathbf{b}-\mathbf{1 m})$ comprising different aryl groups (Scheme 1). For scaffold 2, ten compounds with different groups attached to the indolinone ring were in the HTS library (Figure S3). A hydrogen (8a), an alkyl (8b), an alkyl ketone (8c), a carboxylic acid (9a), or an amide (9b) substituent resulted in no Eis_Mtb inhibitory activity. Similarly to the scaffold $\mathbf{1}$ analogues, aryl ketones of scaffold $\mathbf{2}(\mathbf{8} \mathbf{f}-\mathbf{8 h})$ displayed Eis_M $t b$ inhibition, with the exception of the trifluoromethyl substituted aryl ketones (8d and 8e). Three compounds synthesized from scaffold $\mathbf{2}$ (8f$\mathbf{8 h}$, Figure S3) were found to be active. These and 11 of their

Received: July 7, 2016

Accepted: September 15, 2016

Published: September 15, 2016 
analogues contain different aryl substituents for further study (Note: $\mathbf{8 f} \mathbf{-} \mathbf{8 h}$ are numbered $\mathbf{2 g}-\mathbf{2 i}$ in Scheme 1 and Figure $\mathrm{S} 1$ to represent the scaffold 2 series).

Compound 1a and 12 analogues $(\mathbf{1 b}-\mathbf{1 m})$ as well as 14 analogues for scaffold $\mathbf{2}(\mathbf{2 a}-\mathbf{2 n})$ with different $\mathrm{R}$ substituents were synthesized for structure-activity relationship (SAR) analysis of Eis_Mtb inhibition in vitro and in cellulo (Scheme 1). All new compounds were characterized by ${ }^{1} \mathrm{H},{ }^{13} \mathrm{C}$ NMR (Figures S5-S56), and mass spectrometry and were established to be $\geq 95 \%$ pure by HPLC prior to further testing.

We evaluated biochemical (inhibition $\left(\mathrm{IC}_{50}\right)$ of purified Eis $M t b$ enzyme) and biological (effect on the KAN MIC values for KAN-sensitive $M t b \mathrm{H} 37 \mathrm{Rv}$ and KAN-resistant $M t b$ K204 cells) properties of these compounds, in parallel (Table 1; Figure S57).

Table 1. IC $_{50}$ Values against Purified Eis $M t b$ and MIC Values against $M t b \mathrm{H} 37 \mathrm{Rv}$ and $M t b \mathrm{~K} 204$ with the Compounds at the Concentrations Specified

\begin{tabular}{|c|c|c|c|c|}
\hline compd & $\mathrm{IC}_{50}(\mu \mathrm{M})^{a}$ & $\begin{array}{l}\text { concentration } \\
\text { tested }(\mu \mathrm{M})^{b}\end{array}$ & $\begin{array}{c}\mathrm{H} 37 \mathrm{Rv} \\
\mathrm{MIC}_{\mathrm{KAN}} \\
(\mu \mathrm{g} / \mathrm{mL})^{c}\end{array}$ & $\begin{array}{c}\mathrm{K}^{204 \mathrm{MIC}_{\mathrm{KAN}}} \\
(\mu \mathrm{g} / \mathrm{mL})^{\mathrm{d}}\end{array}$ \\
\hline & & & $\leq 1.25$ & $\geq 10,10$ \\
\hline $1 \mathrm{a}$ & $3 \pm 1$ & 100 & $\leq 1.25$ & 5,10 \\
\hline $1 b$ & $2.9 \pm 0.9$ & 100 & $\leq 1.25$ & 10,10 \\
\hline 1c & $0.16 \pm 0.07$ & 15.5 & $\leq 1.25$ & 10,10 \\
\hline $1 d$ & $0.25 \pm 0.08$ & 25.1 & $\leq 1.25$ & $\geq 10,10$ \\
\hline 1e & $0.5 \pm 0.2$ & 52.6 & $\leq 1.25$ & $\geq 10,10$ \\
\hline If & $>200$ & 100 & $\leq 1.25$ & $\geq 10,10$ \\
\hline $1 \mathrm{~g}$ & $0.3 \pm 0.1$ & 33.9 & $\leq 1.25$ & 10,10 \\
\hline $1 \mathrm{~h}$ & $0.6 \pm 0.3$ & 64.5 & $\leq 1.25$ & $10, \geq 10$ \\
\hline $1 \mathbf{i}$ & $>200$ & 100 & $\leq 1.25$ & $\geq 10, \geq 10$ \\
\hline $\mathbf{1 j}$ & $5.8 \pm 1.8$ & 100 & $\leq 1.25$ & 5,10 \\
\hline $1 \mathrm{k}$ & $>200$ & 100 & $\leq 1.25$ & 10,10 \\
\hline 11 & $>200$ & 100 & $\leq 1.25$ & $\geq 10,10$ \\
\hline $1 \mathrm{~m}$ & $>200$ & 100 & $\leq 1.25$ & $\geq 10,10$ \\
\hline $2 a$ & $0.33 \pm 0.16$ & 32.6 & $\leq 1.25$ & 5,5 \\
\hline $2 b$ & $23 \pm 10$ & 100 & $\leq 1.25$ & 10,10 \\
\hline $2 c$ & $0.30 \pm 0.08$ & 29.7 & $\leq 1.25$ & $5,2.5$ \\
\hline $2 d$ & $0.015 \pm 0.005$ & 1.5 & $\leq 1.25$ & $\geq 10,10$ \\
\hline $2 e$ & $0.54 \pm 0.25$ & 54.2 & $\leq 1.25$ & 10,5 \\
\hline $2 f$ & $>200$ & 100 & $\leq 1.25$ & $\geq 10,10$ \\
\hline $2 \mathrm{~g}$ & $0.09 \pm 0.03$ & 8.9 & $\leq 1.25$ & 5,5 \\
\hline $2 \mathrm{~h}$ & $2.2 \pm 0.7$ & 100 & $\leq 1.25$ & 10,5 \\
\hline $2 \mathrm{i}$ & $>200$ & 100 & $\leq 1.25$ & $\geq 10,10$ \\
\hline $2 j$ & $8.7 \pm 2.2$ & 100 & $\leq 1.25$ & 5,10 \\
\hline $2 k$ & $>200$ & 100 & $\leq 1.25$ & 10,10 \\
\hline 21 & $>200$ & 100 & $\leq 1.25$ & $\geq 10,10$ \\
\hline $2 m$ & $>200$ & 100 & $\leq 1.25$ & $\geq 10,10$ \\
\hline $2 n$ & $1.2 \pm 0.4$ & 100 & $\leq 1.25$ & 10,5 \\
\hline
\end{tabular}

${ }^{a} \mathrm{IC}_{50}$ against purified Eis. ${ }^{b}$ Concentrations of Eis inhibitor in the MIC assays. At these concentrations, these compounds did not inhibit the growth of $M t b \mathrm{H} 37 \mathrm{Rv}$ or that of $M t b \mathrm{~K} 204$ when tested in the absence of KAN. Concentrations of Eis inhibitors were $100 \times$ their $\mathrm{IC}_{50}$ when $\mathrm{IC}_{50}<1 \mu \mathrm{M}$ or $100 \mu \mathrm{M}$ for $\mathrm{IC}_{50}>1 \mu \mathrm{M}$. ${ }^{c}$ Activity of KAN against $M t b$ H37Rv. ${ }^{d}$ Activity of KAN against Mtb K204.

The freshly synthesized 1a, which displayed some inhibition of Eis $M t b$ in the HTS campaign, was confirmed to be a good Eis_Mtb inhibitor in vitro $\left(\mathrm{IC}_{50}=3 \pm 1 \mu \mathrm{M}\right)$. In the presence of $1 \mathrm{a}$, $\mathrm{KAN}$ displayed an MIC of 5-10 $\mu \mathrm{g} / \mathrm{mL}$ against $\mathrm{M} t b \mathrm{~K} 204$. Having confirmed the weak inhibitory activity of $1 \mathrm{a}$, we explored the effect of substitution on the phenyl ring on the aryl ketone part of scaffold $\mathbf{1}$. Ortho substitution, as in $\mathbf{1} \mathbf{b}$ with a $o$-fluoro substituent, resulted in almost the same Eis $M t b$ inhibitory activity $\left(\mathrm{IC}_{50}=2.9 \pm 0.9\right.$ $\mu \mathrm{M})$ as that for the parent 1a. KAN MIC against Mtb K204 was unaffected by $\mathbf{1 b}\left(\mathrm{MIC}_{\mathrm{KAN}}=10 \mu \mathrm{g} / \mathrm{mL}\right)$. To establish if meta or para substitution would be more favorable than ortho substitution, we generated compounds $\mathbf{1} \mathbf{c}-\mathbf{1 j}$. For both meta and para substitutions, bulkier substituents led to weaker Eis $M t b$ inhibition $\left(\mathrm{IC}_{50}>200 \mu \mathrm{M}\right.$ for $m$-methoxy (1f) and $p$-bromo (1i) compared to $\mathrm{IC}_{50}=0.16 \pm 0.07$ and $0.3 \pm 0.1 \mu \mathrm{M}$ for $m$-fluoro (1c) and $p$-fluoro (1g), respectively). Most of these derivatives $(\mathbf{1 c}-\mathbf{1 i})$ did not improve KAN activity against Mtb K204. The $p$ methyl derivative $\mathbf{1 j}$ displayed almost the same Eis_Mtb inhibitory activity $\left(\mathrm{IC}_{50}=5.8 \pm 1.8 \mu \mathrm{M}\right)$ and MIC value against $M t b \mathrm{~K} 204$ as that of $1 \mathbf{a}$. We generated $1 \mathbf{k}$ (naphthyl substituted) with the hope of strengthening any possible $\pi-\pi$ interactions between the inhibitor and the AG-binding site of the $M t b$ Eis. This compound was found to be completely inactive $\left(\mathrm{IC}_{50}>200 \mu \mathrm{M}\right.$ and $\mathrm{MIC}_{\mathrm{KAN}}=$ $10 \mu \mathrm{g} / \mathrm{mL}$ against $M t b \mathrm{~K} 204)$. Finally, replacing the phenyl ring with alkyl chains (ethyl (11) and $t$-butyl (1m)) did not improve the Eis inhibition or $\mathrm{MIC}_{\mathrm{KAN}}$ for K204 $\mathrm{M} t b$.

For scaffold 2, compounds $2 \mathrm{~g}-\mathbf{2} \mathbf{i}$, which displayed Eis $M t b$ inhibition in the HTS, were freshly synthesized. The $p$-fluoro substituted $2 \mathrm{~g}$ displayed good Eis inhibitory activity $\left(\mathrm{IC}_{50}=0.09 \pm\right.$ $0.03 \mu \mathrm{M})$ and, when used in combination with KAN, resulted in $\mathrm{MIC}_{\mathrm{KAN}}$ of $5 \mu \mathrm{g} / \mathrm{mL}$ against $\mathrm{K} 204 \mathrm{Mtb}$. The $p$-chloro substituted $2 \mathrm{~h}$ was less active $\left(\mathrm{IC}_{50}=2.2 \pm 0.7 \mu \mathrm{M}\right)$ than the $p$-fluoro substituted $2 \mathrm{~g}$ and did not sensitize $M t b \mathrm{~K} 204$ to $\mathrm{KAN}\left(\mathrm{MIC}_{\mathrm{KAN}}=\right.$ 5-10 g/mL). The $p$-bromo substituted $2 \mathrm{i}$ was found to be completely inactive $\left(\mathrm{IC}_{50}>200 \mu \mathrm{M}\right.$ and $\mathrm{MIC}_{\mathrm{KAN}} \geq 10 \mu \mathrm{g} / \mathrm{mL}$ against Mtb K204), while it displayed limited Eis inhibition in the HTS, which could indicate that the compound in the HTS library was not completely pure. We also found that the $p$-methyl derivative $2 \mathrm{j}$ displayed a 100 -fold decrease in Eis inhibitory activity $\left(\mathrm{IC}_{50}=8.7 \pm 2.2 \mu \mathrm{M}\right.$ ) from $2 \mathrm{~g}$ and, in combination with $\mathrm{KAN}$, resulted in almost the same KAN MIC $(5-10 \mu \mathrm{g} / \mathrm{mL})$ against KAN-resistant $M t b$ as $\mathbf{2 g}$ did. The nonsubstituted counterpart of parent $\mathbf{2 g}$, derivative $\mathbf{2 a}$, displayed weaker Eis inhibitory activity $\left(\mathrm{IC}_{50}=0.33 \pm 0.16 \mu \mathrm{M}\right)$ and improved the activity of KAN against $M t b \mathrm{~K} 204\left(\mathrm{MIC}_{\mathrm{KAN}}=5 \mu \mathrm{g} / \mathrm{mL}\right)$. We also synthesized the $m$ fluoro, $m$-chloro, and $m$-bromo derivatives $2 \mathrm{c}$, $2 \mathrm{~d}$, and $2 \mathrm{e}$. The $m$ chloro substituted $\mathbf{2 d}$ showed the same inhibitory activity as that of $2 \mathrm{~g}$ but did not sensitize $M t b \mathrm{~K} 204$ to $\mathrm{KAN}\left(\mathrm{MIC}_{\mathrm{KAN}} \geq 10 \mu \mathrm{g} / \mathrm{mL}\right)$. The $m$-fluoro and $m$-bromo substituted $2 \mathrm{c}$ and $2 \mathbf{e}$ resulted in a 3and 5-fold worse Eis inhibitory activity $\left(\mathrm{IC}_{50}=0.30 \pm 0.08\right.$ and 0.54 $\pm 0.25 \mu \mathrm{M})$, respectively. When used with the $m$-bromosubstituted 2e, KAN had a MIC of 5-10 $\mu \mathrm{g} / \mathrm{mL}$ against $M t b$ K204. However, when used with the $m$-fluoro substituted 2c, KAN displayed a better MIC value of $2.5-5 \mu \mathrm{g} / \mathrm{mL}$. As observed with scaffold 1, the presence of $m$-methoxy-phenyl, naphthyl, ethyl, and $t$-butyl groups in scaffold 2 resulted in molecules that were completely inactive $\left(\mathrm{IC}_{50}>200 \mu \mathrm{M}\right.$ and $\mathrm{MIC}_{\mathrm{KAN}} \geq 10 \mu \mathrm{g} / \mathrm{mL}$ against $M t b$ K204). For scaffold 2, we also synthesized a $m$-nitro substituted compound (2n) to investigate the potential effect of a strong electron-withdrawing group on Eis inhibitory activity. Compound $2 \mathrm{n}$ was less active $\left(\mathrm{IC}_{50}=1.2 \pm 0.4 \mu \mathrm{M}\right)$ than $2 \mathrm{~g}$, but it sensitized $M t b \mathrm{~K} 204$ to $\mathrm{KAN}\left(\mathrm{MIC}_{\mathrm{KAN}}=5-10 \mu \mathrm{g} / \mathrm{mL}\right)$. The absence of antibacterial activity of these compounds when used alone along with a general correlation between $\mathrm{IC}_{50}$ and $\mathrm{MIC}$ values indicated that inhibition of Eis by these compounds is the main mechanism of sensitization to KAN.

To investigate the selectivity of our inhibitors toward Eis $M t b$, we tested two of our derivatives, one from each series, $\mathbf{1 c}$ and $\mathbf{2 c}$, against three other AAC enzymes: $\mathrm{AAC}\left(2^{\prime}\right)-\mathrm{Ic},{ }^{4} \mathrm{AAC}(3)-\mathrm{IV},{ }^{8}$ and $\mathrm{AAC}\left(6^{\prime}\right)-\mathrm{Ie} / \mathrm{APH}\left(2^{\prime \prime}\right)-\mathrm{Ia}^{8}{ }^{8}$ Similarly to other known non-Eis 
AACs, these three enzymes were previously shown to be strictly regiospecific, but like Eis, each enzyme was capable of acetylating structurally distinct AGs. Neither 1c nor 2c inhibited KAN acetylation by these three AACs at concentrations as high as 200 $\mu \mathrm{M}$, which indicated that our compounds were highly selective against Eis $M t b$.

To explain the results of our SAR study, we used previously published crystal structures of ternaryEis_Mtb-CoA-compound A (13g in ref 9; PDB ID 5EC4) and $\mathbf{B}$ (11) in ref 9; PDB ID 5EBV) complexes to model our inhibitors $1 \mathrm{a}$ and $\mathbf{2 g}$ in a position similar to that of inhibitors A and B (Figure S4). Without the crystal structures, de novo computational modeling of and screening for Eis inhibitors, including pharmacophore-based computer modeling, are invalidated by significant conformational changes in the Eis active site upon inhibitor binding. ${ }^{9}$ The inhibitors occupy the site overlapping with the AG-binding site of Eis. The models show that the cores of inhibitors $\mathbf{1 a}$ and $\mathbf{2 g}$ are surrounded by the side chains of hydrophobic amino acid residues (Trp36, the aliphatic part of Glu401, Ile28, Phe24, and Val400). The cores stack with the indole of $\operatorname{Trp} 36$, and in the orthogonal direction they are sandwiched between Glu401, the Eis C-terminus on one side and Ile 28 on the other. The acetophenone rings of both series 1 and $\mathbf{2}$ with different substituents stack with Phe84, explaining why replacing these aromatic rings with alkyl chains resulted in a loss of activity for $\mathbf{1 1}$, $\mathbf{1 m}, \mathbf{2 l}$, and $\mathbf{2 m}$. The acetophenone rings are also surrounded by several hydrophobic amino acid residues (Phe84, Trp36, Met65, Ala33). Thus, putting a polar methoxy group in this hydrophobic environment would likely destabilize Eis binding, explaining the $\mathrm{IC}_{50}$ values of $>200 \mu \mathrm{M}$ for $\mathbf{1 f}$ and $2 \mathrm{f}$. The para position of the acetophenone rings is flanked by Phe 84 and Trp36, and it is $~ 5 \AA$ away from Trp 13 and Met65, explaining why the bulkier bromo substituents of $\mathbf{1 i}$ and $\mathbf{2} \mathbf{i}$ resulted in lower Eis inhibitory activity, whereas the small fluoro substituents of $\mathbf{1 g}$ and $\mathbf{2 g}$ improved Eis inhibitory activity. The ortho position of the acetophenone rings is flanked by Phe402, explaining why an ortho substituent, as in $\mathbf{1 b}$ and $\mathbf{2 b}$, resulted in a loss of Eis inhibition. The models shows that there is space for small substitution in the meta position of the acetophenone rings (a $5 \AA$ gap). Small substituents such as the fluoro and chloro of $\mathbf{1 c}, \mathbf{1 d}, \mathbf{2 c}$, and $\mathbf{2 d}$ fit well in the cavity, explaining why these compounds displayed good Eis inhibition. However, bulkier substituents such as the bromo of $1 \mathbf{e}$ and $\mathbf{2 e}$ or the nitro of $2 \mathrm{n}$ are too big to be accommodated at this site and would clash with Eis residues, accounting for the poor Eis inhibition by these compounds. We also determined that the calculated $\log P$ values of all compounds are in the desirable range (0.98-3.75; Table S1).

In sum, we have discovered two scaffolds with Eis inhibitory activity. From 27 synthesized analogues of these scaffolds with the variable acetophenone appendage, we identified potent inhibitors of Eis. Growth inhibition studies of our inhibitors in combination with $\mathrm{KAN}$ in $\mathrm{KAN}$-susceptible $\mathrm{M} t b \mathrm{H} 37 \mathrm{Rv}\left(\mathrm{MIC}_{\mathrm{KAN}} \leq 1.25 \mu \mathrm{g} /\right.$ $\mathrm{mL})$ and KAN-resistant $\mathrm{M} t b \mathrm{~K} 204\left(\mathrm{MIC}_{\mathrm{KAN}} \geq 10 \mu \mathrm{g} / \mathrm{mL}\right)$ showed that some of our inhibitors were able to sensitize $M t b$ K204 to KAN. Smaller substituents, like hydrogen and fluorine, yielded the best compounds. In contrast, larger substituents, such as bromo or methoxy dramatically decreased the potency of the compounds. The best compound identified was $2 \mathrm{c}$ with the 3 - (1,3dioxolano)-2-indolinone core and a $m$-fluoro-phenyl substituent. This compound when used in combination with KAN reduced the $\mathrm{MIC}_{\mathrm{KAN}}$ for KAN-resistant $M t b$ to $2.5-5 \mu \mathrm{g} / \mathrm{mL}$. While CLSI recommends $\mathrm{MIC}_{\mathrm{KAN}}$ of $5 \mu \mathrm{g} / \mathrm{mL}$ on Middlebrook $7 \mathrm{H} 10$ agar, it has no recommendation for susceptibility testing by Alamar Blue. One study suggests a critical MIC $\mathrm{KAN}_{\mathrm{N}}$ of $2.5 \mu \mathrm{g} / \mathrm{mL}$ for Alamar Blue testing. Since our inhibitors are able to return KAN-resistant isolates to an $\mathrm{MIC}_{\mathrm{KAN}}$ below the critical concentration, essentially making resistant $M t b$ isolate $\mathrm{KAN}$-susceptible, such inhibitors could play a crucial role in recovering KAN as a treatment option. However, clinical studies to support this hypothesis are yet to be undertaken. We are actively pursuing these avenues.

\section{ASSOCIATED CONTENT}

\section{Supporting Information}

The Supporting Information is available free of charge on the ACS Publications website at DOI: 10.1021/acsmedchemlett.6b00261.

Structures, models, experimental procedures, and characterization data (PDF)

\section{AUTHOR INFORMATION}

\section{Corresponding Author}

*(S.G.-T.) E-mail: sylviegtsodikova@uky.edu. Phone: 859-2181686. Fax: 859-257-7585. (J.E.P.) E-mail: jposey@cdc.gov.

\section{Funding}

This work was supported by a NIH Grant AI090048 (S.G.-T.), a grant from the CCG at the U. Michigan (S.G.-T.), a grant from the Firland Foundation (S.G.-T.), and by startup funds from the College of Pharmacy at the U. Kentucky (S.G.-T. and O.V.T.). We thank Steve Vander Roest, Martha Larsen, and Paul Kirchhoff (CCG, UM) for help with HTS.

\section{Notes}

The authors declare no competing financial interest.

\section{REFERENCES}

(1) Zaunbrecher, M. A.; Sikes, R. D., Jr.; Metchock, B.; Shinnick, T. M.; Posey, J. E. Overexpression of the chromosomally encoded aminoglycoside acetyltransferase eis confers kanamycin resistance in Mycobacterium tuberculosis. Proc. Natl. Acad. Sci. U. S. A. 2009, 106, 20004-20009.

(2) Chen, W.; Green, K. D.; Tsodikov, O. V.; Garneau-Tsodikova, S. Aminoglycoside multiacetylating activity of the enhanced intracellular survival protein from Mycobacterium smegmatis and its inhibition. Biochemistry 2012, 51, 4959-4967.

(3) Houghton, J.L.; Green, K. D.; Pricer, R. E.; Mayhoub, A. S.; GarneauTsodikova, S. Unexpected $\mathrm{N}$-acetylation of capreomycin by mycobacterial Eis enzymes. J. Antimicrob. Chemother. 2013, 68, 800-805.

(4) Chen, W.; Biswas, T.; Porter, V. R.; Tsodikov, O. V.; GarneauTsodikova, $\mathrm{S}$. Unusual regioversatility of acetyltransferase Eis, a cause of drug resistance in XDR-TB. Proc. Natl.Acad.Sci.U.S.A. 2011, 108, 98049808.

(5) Green, K. D; Pricer, R. E.; Stewart, M. N.; Garneau-Tsodikova, S. Comparative study of Eis-like enzymes from pathogenic and nonpagthogenic bacteria. ACS Infect. Dis. 2015, 1, 272-283.

(6) Green, K. D.; Biswas, T.; Chang, C.; Wu, R.; Chen, W.; Janes, B. K.; Chalupska, D.; Gornicki, P.; Hanna, P.C.; Tsodikov, O.V.;Joachimiak, A.; Garneau-Tsodikova, S. Biochemical and structural analysis of an Eis family aminoglycoside acetyltransferase from Bacillus anthracis. Biochemistry 2015, 54, 3197-3206.

(7) Green, K. D.; Chen, W.; Garneau-Tsodikova, S. Identification and characterization of inhibitors of the aminoglycoside resistance acetyltransferase Eis from Mycobacterium tuberculosis. ChemMedChem 2012, 7, $73-77$.

(8) Green, K. D.; Chen, W.; Houghton, J. L.; Fridman, M.; GarneauTsodikova, S. Exploring the substrate promiscuity of drug-modifying enzymes for the chemoenzymatic generation of $\mathrm{N}$-acylated aminoglycosides. ChemBioChem 2010, 11, 119-126.

(9) Willby, M. J.; Green, K. D.; Gajadeera, C. S.; Hou, C.; Tsodikov, O. V.; Posey, J. E.; Garneau-Tsodikova, S. Potent inhibitors of acetyltransferase Eis overcome kanamycin resistance in Mycobacterium tuberculosis. ACS Chem. Biol. 2016, 11, 1639-1646. 